\title{
Quality of Care in Rheumatoid Disease from the Clinician Perspective: A Modified Delphi Panel Approach
}

\author{
Finbar O'Shea · Peter C. Taylor - Gavin Dickie · Andrew Yaworsky • \\ Benjamin Banderas · Sumesh Kachroo
}

Received: February 14, 2018 / Published online: April 3, 2018

(C) The Author(s) 2018

\section{ABSTRACT}

Introduction: To establish clinical consensus on important and relevant quality-of-care (QoC) attributes in rheumatic disease (RD) treatment that may improve treatment outcomes and guide best practices.

Methods: Twenty-three QoC attributes were identified in a literature review. Fifteen European-based clinicians were selected based on their contributions to RD guidelines, publications, and patient care. A three-round (an interview round and two web-based rounds)

Enhanced content To view enhanced content for this article go to https://doi.org/10.6084/m9.figshare. 5967031.

F. O'Shea

St. James's Hospital, Dublin, Ireland

P. C. Taylor

Nuffield Department of Orthopaedics, Rheumatology and Musculoskeletal Sciences, Botnar Research Centre, University of Oxford, Oxford, UK

G. Dickie · A. Yaworsky · B. Banderas $(\bowtie)$

Adelphi Values LLC, Boston, MA, USA

e-mail: benjamin.banderas@adelphivalues.com

S. Kachroo

Center for Observational and Real-World Evidence

(CORE), Merck \& Co., Inc., Kenilworth, NJ, USA modified Delphi panel was conducted to reach consensus and finalize a QoC attribute list.

Results: In round 1 (clinician interviews), clinicians reported 52 unique QoC attributes across 14 themes, with the greatest number of attributes reported in the "treatment goals" $(n=7)$ and "remote monitoring" $(n=7)$ themes. During rounds 2 and 3, the critically important QoC attributes most frequently reported were access to care/treatment $(n=14$, $93.3 \%$ ), safety of treatment (round $2 n=14$, $93.3 \%$, round $3 n=13,86.7 \%$ ), and access to clinicians and specialists (round 2: $n=13$, $86.7 \%$, round 3: $n=14,93.3 \%)$. The final list contained 53 QoC attributes.

Conclusion: The study demonstrates consensus across several themes of QoC. Quality of care is a complex, multidimensional, and fluid concept that can be improved by ensuring patients have access to care, open communication between patients and clinicians, and the use of novel strategies, such as remote monitoring. Utilization of the attribute list can potentially improve the lives of patients, provide clinicians with tools to provide greater QoC, and improve the healthcare system as a whole.

Funding: Merck \& Co., Inc.

Keywords: Ankylosing spondylitis; Clinicians; Psoriatic arthritis; Quality of care; Rheumatoid arthritis; Rheumatoid disease; Treatment 


\section{INTRODUCTION}

Rheumatic diseases (RD) envelops several chronic and disabling autoimmune disorders [1-5] that affect the joints and connective tissue of patients, including rheumatoid arthritis (RA) [1], ankylosing spondylitis (AS) [2], and psoriatic arthritis (PsA) [6]. Beyond the symptoms, $\mathrm{RD}$ can lead to a significant burden on patient quality of life [3, 7-10].

Despite the available treatments [11-14] and key treatment guidelines [15-17], the associated outcomes are not always ideal; a gap remains between health-system expenditure and positive treatment outcomes [18-20]. In order to improve treatment outcomes, it is imperative to understand the attributes of quality of care (QoC) that clinicians utilize when making treatment decisions regarding patients with RD.

Broadly, QoC refers to "the degree to which health services for individuals and populations increase the likelihood of desired health outcomes and are consistent with current professional knowledge" [21]. QoC involves the provision of safe, efficacious, patient-centric, and equitable treatment to patients that achieves desired treatment goals in a timely and efficient manner [21]. Quality of care is of particular importance in $\mathrm{RD}$ due to its chronic nature, wide-ranging symptomology [1-5], and associated patient burden $[7,9,10,22]$. To achieve remission and avoid long-term damage and impact on quality of life, treatment plans must be tailored to the needs and characteristics of individual patients [23], a consideration that is counter to the "one-size-fits-all" tendency within existing RD treatment guidelines [15-17].

Despite a need for a greater understanding of QoC metrics in RD, there is limited evidence available. Previous work has suggested patient-clinician communication, access to treatment, and treatment efficacy are key factors influencing QoC [24]. Other work has indicated that coordination among relevant specialists and consideration of individual patient characteristics can improve QoC [25-27]. However, the evolving nature of QoC contributes to the lack of clarity regarding the attributes that clinicians consider critical. The current study aimed at achieving consensus around the attributes of QoC using a Delphi panel approach to guide future RD research (with a specific focus on RA, AS, and PsA) and best practices across Europe.

\section{METHODS}

The consensus process utilized a three-round modified Delphi panel (MDP) approach: an interview round with five clinicians and two web-based rounds with 15 clinicians (Fig. 1).

Clinician feedback on the QoC attribute list was collected, implemented, and then returned to the panelists for additional comment [28]. All experts were blinded to one another to avoid bias. As this study involved clinician participants providing information on QoC in RD, rather than information on themselves, independent review board approval was not deemed necessary. However, experts provided consent before participation, in line with best practice.

\section{Panel Selection}

The 15 Europe-based clinicians selected for participation met at least one of the following criteria: (1) currently treat patients with RD (i.e., RA, AS, and PsA), (2) influence broader guideline-oriented decisions for RD (i.e., the development of current and/or historical treatment guidelines), and/or (3) are well published within the area of RD. Eligible clinicians were identified by reviewing the literature and current and historical RD treatment guideline author lists.

\section{Draft Quality-of-Care Attribute List}

Three targeted reviews of published literature were conducted in March 2016 using the OvidSP platform, one for each $\mathrm{RD}$ condition (i.e., RA, AS, PsA). Search strategies included terms for the conditions (i.e., "rheumatoid arthritis," "ankylosing spondylitis," "psoriatic arthritis") AND terms related to QoC (e.g., "quality of care," "health care quality," "quality improvement," etc.) AND terms related to 


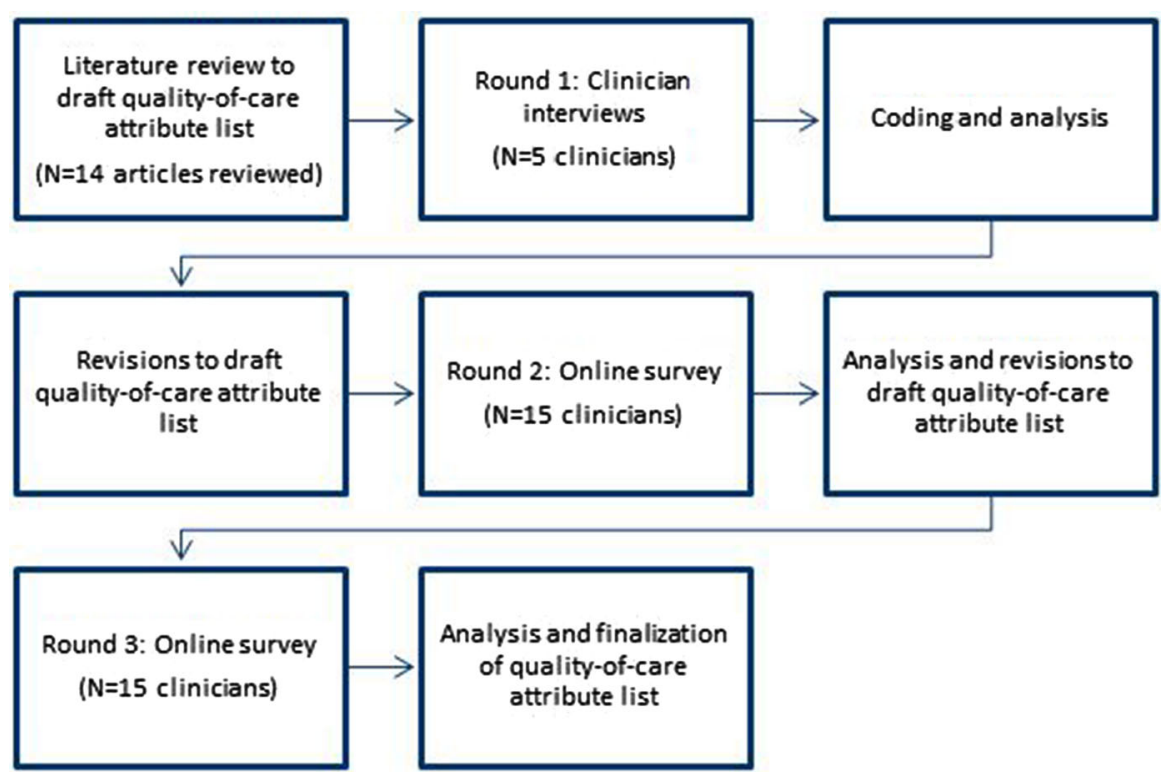

Fig. 1 Overview of the study methodology

treatment-related decision-making (i.e., "decision making," "treatment-related decision making," etc.). Results were used to compile a preliminary list of QoC attributes.

\section{Round 1}

Individual 60-min telephone interviews were conducted with five European RD clinicians. Trained interviewers used a semi-structured interview guide with open-ended questioning to prompt clinicians to identify and describe, in their own words, QoC attributes they considered important when making treatment-related decisions in RD care. Targeted probes were subsequently used to obtain specific information on attributes of interest. In addition, clinicians were asked to provide feedback on the preliminary attribute list.

Interviews were audio-recorded (with clinician consent), anonymized, and transcribed. Analysis entailed identifying and coding individual attributes reported by clinicians. Unique QoC attributes were tabulated by frequency, and descriptions were developed based on clinician language. Round 1 results were used to revise the QoC attribute list for round 2. Attributes were further grouped by theme, based on content analysis; attributes considered to constitute a larger conceptual idea were listed under a single heading (e.g., access to care and access to information are both considered part of the broader theme of "accessibility"). Clinician consensus on theme organization was not considered an objective of this study, and the QoC attribute list was presented to clinicians in round 2 and round 3 without themes identified.

\section{Round 2}

The QoC attribute list was ported to a digital format (SurveyMonkey ${ }^{\mathrm{TM}}$ ), and a link was emailed to the panel of 15 clinicians with instructions for completing the MDP. Clinicians viewed the attribute list electronically and rated the concepts according to their perceived importance (options included "critically important," "important but not critical," "somewhat important," and "not at all important"). Open fields were included at the end of the list for clinicians to provide qualitative feedback (e.g., suggestion that attributes be removed or merged, or new attributes added).

The results of round 2 were analyzed utilizing descriptive statistics. All data-the QoC attributes and their associated ratings-were 
tabulated, and frequency counts for each rating option were generated. The results of the analysis were used to revise the list of attributes. Attributes reported to be "somewhat important" and/or "not at all important" by $>33.3 \%$ of clinicians during the round (i.e., $n>5$ ) were considered for removal. Attributes reported as both "critically important" and "important but not critical," but also meeting the guideline for removal, were reviewed for content and were less likely to be removed. For example, an attribute rated as "critically important" by nine clinicians (60.0\%), "somewhat important" by five clinicians (33.3\%), and "not important at all" by one clinician (6.7\%), the item would still be considered for retention, as all but one clinician reported the concept to be "important." Additional attributes were added if $>33.3 \%$ of clinicians $(n>5)$ suggested them during round 2 .

\section{Round 3}

The revised QoC attribute list was sent to the 15 experts, who repeated the process outlined in round 2 . The same descriptive statistics analysis used in round 2 was performed on the data, and the QoC attribute list was finalized based on the results.

\section{RESULTS}

\section{Sample}

All clinicians who participated in the MDP were based in Europe, including Germany and Spain ( $n=3$ each); the Netherlands $(n=2)$; and Belgium, Czech Republic, Greece, Hungary, Ireland, Serbia, and the United Kingdom $(n=1$ each). Most of the clinicians specialized in general rheumatology $(n=13,86.7 \%)$, the majority reported their practice type to be a teaching hospital $(n=12,80.0 \%)$, and most (60.0\%) reported a practice size of 6-20 clinicians. The majority $(n=8,53.3 \%)$ had 20 or more years of experience practicing medicine, and most had been involved in the development of RD treatment guidelines $(n=11,73.3 \%)$.

\section{Literature Review}

Of the 300 abstracts screened, 14 articles were selected for full review. The 23 attributes identified from the literature review comprised the initial QoC attribute list presented in round 1.

\section{Round 1}

A total of 52 unique QoC attributes, across 14 themes, were reported. The greatest number of attributes were classified in the "treatment goals" and "remote monitoring" themes $(n=7)$. All clinicians $(n=5,100.0 \%)$ identified access to care, access to information, patient involvement in decision-making, communication between patients and clinicians, ensuring that patients understand disease and treatment, coordination of treatment-based care among clinicians, monitoring in general, considering patient treatment preference, and an individualized treatment plan for patient care as important attributes for QoC.

Round 1 data informed revisions to the draft QoC attribute list provided to experts in round 2 , which comprised 53 attributes across 14 themes.

\section{Round 2}

The critically important QoC attributes most frequently reported by clinicians $(\geq 80.0 \%)$ were access to care/treatment, safety of treatment, and treatment adherence $(n=14,93.3 \%$ each); access to clinicians and specialists, treatment initiation, monitoring of treatment efficacy, and impact on activities of daily living ( $n=13, \quad 86.7 \%$ each); and communication between patients and clinicians, reduction of complications of disease, treatment efficacy, and clinicians being up to date with current treatments and disease management approaches ( $n=12,80.0 \%$ each). The important, but not critical, QoC attributes most frequently reported $(\geq 66.7 \%)$ were ensuring that family/ significant others understand disease and 
treatment ( $n=13,86.7 \%)$; as well as access to supportive services, patient care, and counseling; active patient involvement in decisionmaking; and patient self-monitoring $(n=10$, $66.7 \%$ each). Only one attribute was rated as "somewhat important" by more than half of the clinicians: patient access to remote monitoring technology $(n=8,53.3 \%)$. Only two of the 53 attributes presented in round 2 were rated "not at all important," each by only one clinician (6.7\%): cost to implement remote monitoring technology and understanding patient characteristics.

Figure 2 presents clinicians' ratings for QoC attributes $(n=31)$ reported as "critically important" by $\geq 53.3 \%$ of clinicians $(n \geq 8)$.

\section{Revisions to the Quality-of-Care Attribute List} While no attributes were removed following round 2, minor changes were made to the labels of some QoC attributes. Additional text was added to the attributes "maintain patient's privacy," "understanding patient characteristics," and to the remote monitoring concepts in order to clarify these attributes. No new concepts were added to the QoC attribute list-only three clinicians (20.0\%) suggested adding a new concept, with no more than one (6.7\%) suggesting each specific attribute.

\section{Round 3}

The critically important QoC attributes most frequently reported $(\geq 80.0 \%)$ were: access to care/treatment, access to clinicians and specialists, and treatment efficacy $(n=14,93.3 \%$ each); treatment initiation, safety of treatment, and impact on activities of daily living $(n=13$, $86.7 \%$ each); and communication between patients and clinicians, improved quality of life, and monitoring of treatment efficacy $(n=12$, $80.0 \%$ each). The important, but not critical, QoC attributes most frequently reported ( $\geq 66.7 \%$ ) were access to supportive services, patient care, and counseling; access to information/educational materials; active patient

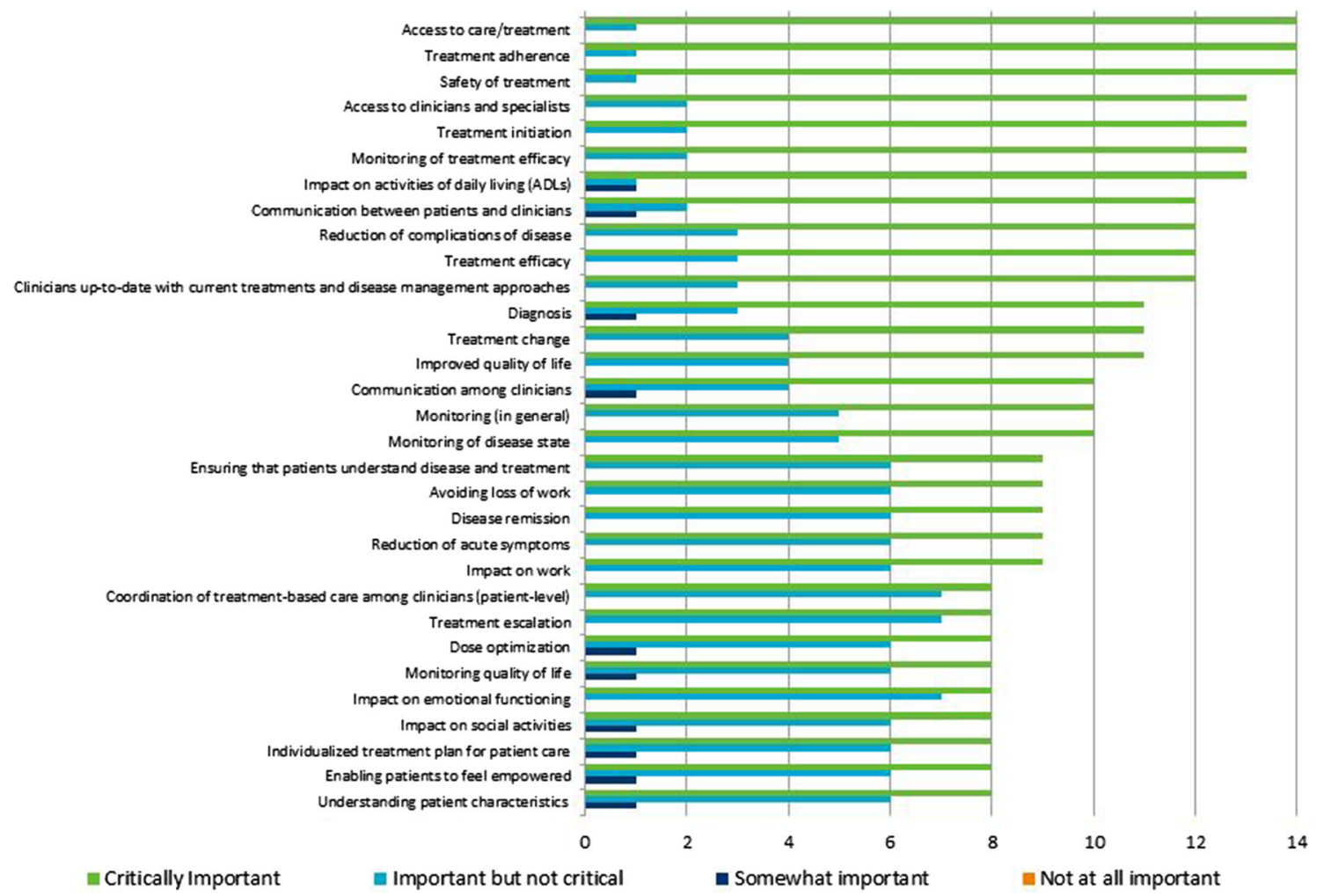

Fig. 2 Round 2 quality-of-care attribute ratings for the most critical attributes $(n=30)$ 
involvement in decision-making; ensuring that family/significant others understand disease and treatment; cost to hospital/facility; monitoring quality of life; and considering patient treatment preference $(n=10,66.7 \%$ each). Only two attributes were rated "somewhat important" by over half of the clinicians: patient ability to use remote monitoring technology $(n=9,60.0 \%)$ and patient access to remote monitoring technology $(n=8,53.3 \%)$. Only two of the 53 attributes presented in round 2 were rated "not at all important," each by only one clinician (6.7\%): holistic treatment approaches and cost to implement remote monitoring technology.

Figure 3 presents clinicians' ratings for QoC attributes $(n=30)$ reported as "critically important" by $\geq 53.3 \%$ of clinicians $(n \geq 8)$.

No changes were made to the QoC attribute list following round 3. The final QoC attribute list (Table 1) consisted of 53 attributes, with access to care/treatment, access to clinicians and specialists, communication between patients and clinicians, treatment initiation, improved quality of life, safety of treatment, treatment efficacy, monitoring treatment efficacy, impact on activities of daily living, and clinicians being up to date with current treatments and disease management approaches reported as being critically important to QoC in $\mathrm{RD}$ by over three-quarters of clinicians.

\section{DISCUSSION}

Improving QoC has been at the forefront of work by both clinicians and healthcare researchers for several decades [29], with a particular focus on defining and measuring such improvement [30, 31]. Whilst QoC has been defined in a disease-nonspecific setting [21], a better understanding of QoC specific to RD may ultimately lead to more efficacious, efficient, and cost-effective methods of delivering patient care. Research has begun to explore QoC in RD in order to improve patient outcomes [25-27].

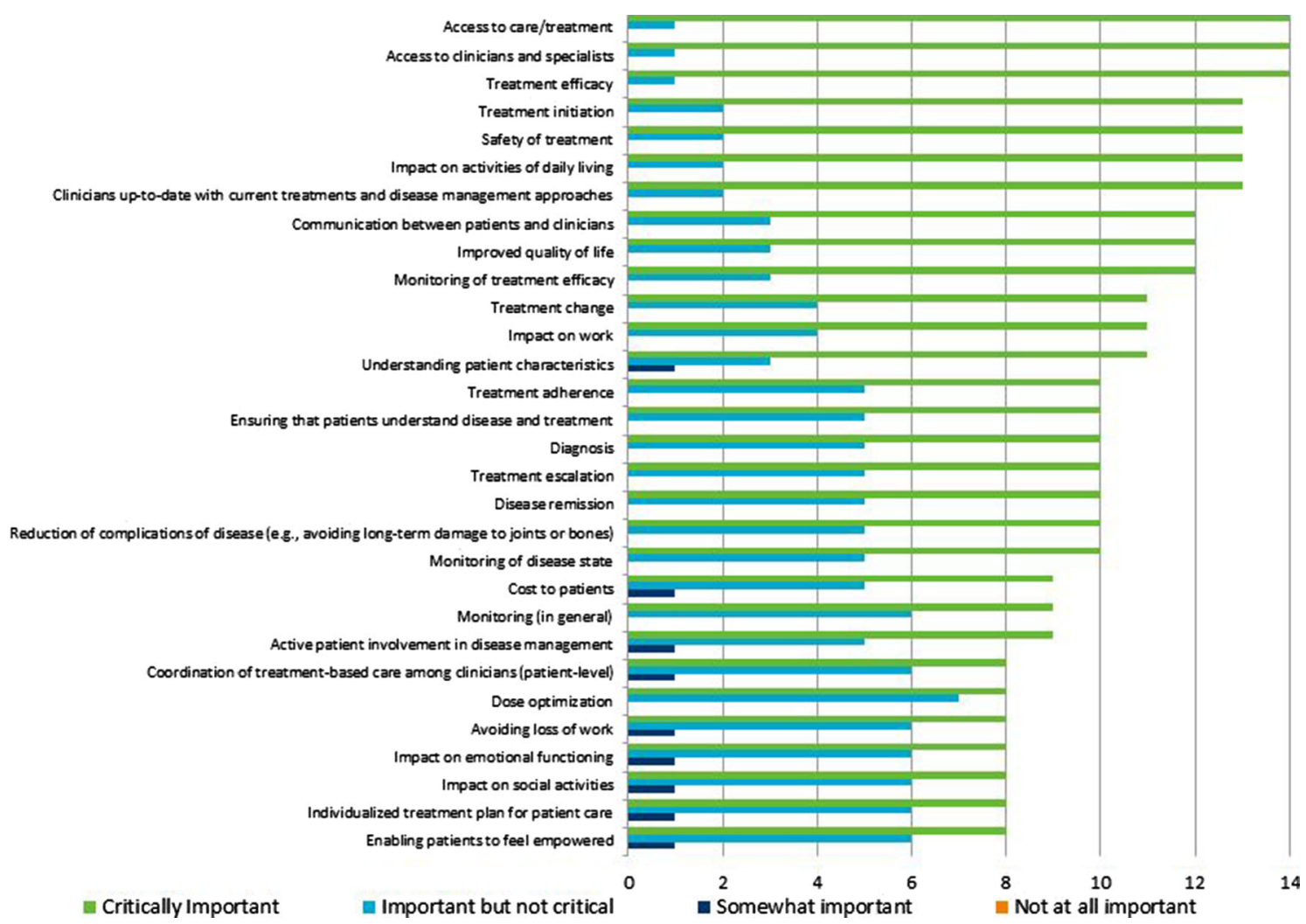

Fig. 3 Round 3 quality-of-care attribute ratings for the most critical attributes $(n=30)$ 
Table 1 Final quality-of-care attribute list $(n=53)$

\section{Attributes}

\section{Accessibility}

Access to care/treatment

Access to clinicians and specialists

Access to supportive services, patient care, and counseling

Access to information/educational materials

\section{Treatment adherence}

Treatment adherence

\section{Communication}

Active patient involvement in decision making

Communication among clinicians

Ensuring that patients understand disease and treatment

Ensuring that family/significant others understand disease and treatment

Communication between patients and clinicians

Communication between clinicians and payers

\section{Coordination of treatment-based care among} clinicians

Coordination of treatment-based care among clinicians (patient level)

\section{Cost of rheumatic disease management}

Cost to hospital/facility

Cost to patients

\section{Disease management}

Diagnosis

Treatment initiation

Treatment escalation

Dose optimization

Treatment change

Holistic treatment approaches

\section{Treatment goals}

Avoiding loss of work

Disease remission
Table 1 continued

\section{Attributes}

Identification of treatment targets

Improved quality of life

Reduction of complications of disease (e.g., avoiding long-term damage to joints or bones)

Safety of treatment

Reduction of acute symptoms

Treatment efficacy

Use of pre-existing guidelines

Treatment guidelines

\section{Monitoring}

Monitoring (in general)

Monitoring of disease state

Patient self-monitoring

Monitoring quality of life

Monitoring of treatment efficacy

\section{Remote monitoring}

Remote monitoring (in general)

Maintaining patient's privacy (e.g., protection of patient medical data and personal information)

Patient access to remote monitoring technology (e.g., e-mail, computers, smartphone applications)

Patient ability to use remote monitoring technology (e.g., email, computers, smartphone applications)

Cost to implement remote monitoring technology

Patient willingness to participate in remote monitoring

Appropriate staff/resources to manage remote monitoring system

\section{Burden on patient and others}

Impact on activities of daily living

Impact on emotional functioning

Impact on family

Impact on social activities

Impact on work 
Table 1 continued

Attributes

Patient centricity

Considering patient treatment preference

Individualized treatment plan for patient care

Active patient involvement in disease management

Understanding patient characteristics

Enabling patients to feel empowered

Clinician training/education

Clinicians up to date with current treatments and disease management approaches

Use of technology

Use of technology (e.g., communication between clinicians, digital access to patient medical records, advanced diagnostic tools)

Less work has focused on identifying individual attributes that contribute to QoC in $\mathrm{RD}$ and their relative importance. The aim of this study was to establish consensus on the QoC attributes considered to be important and when making treatment-related decisions in RD.

Results suggest that key treatment goals for RD patients (specifically RA, AS, and PsA patients) involve providing an efficacious treatment with minimal side effects that reduces complications of the disease (such as longterm joint or bone damage) and allowing patients to achieve and maintain disease remission, thus reducing the burden on patients and improving their quality of life. These treatment goals can be achieved in a number of ways, but paramount is ensuring that patients have easy and direct access to care and clinicians/specialists with knowledge of the most current treatment and disease-management approaches. Being able to access effective care is critical throughout the course of disease, as it allows for timely diagnosis and subsequent initial treatments, as well as changes to disease management (e.g., treatment initiation and escalation) as the disease progresses. Similarly, regularly monitoring treatment efficacy and overall disease state is important as it helps to inform timely changes to disease management.

Following the theme of access over the course of $\mathrm{RD}$, clinicians also stressed the importance of having an open line of communication with patients. Specifically, clinicians noted that open communications can help patients and clinicians work together to consider the individual needs and characteristics of each patient, and to ensure that patients understand their disease and the associated treatment. Clinicians suggested that a patient-clinician collaborative approach to disease management can help promote treatment adherence, as patients who are well informed and feel that their individual preferences have been considered may be more likely to comply with the treatment regimen prescribed by the clinician. Despite the acknowledged importance of open and empathetic communication between patients and their healthcare providers, it may not be feasible for clinicians to partake in this approach due to limited time during patient visits and/or a lack of available supportive resources, which can result in lessthan-ideal disease management.

Remote monitoring of patients outside of clinical visits could be a useful tool for improving patient-clinician communication and overall QoC, and for assessing RD treatment outcomes. Remote monitoring is broadly defined as the use of communication technology [32] to facilitate the assessment of, and patient-clinician discussions around, a patient's disease state, treatment efficacy, and patient satisfaction (e.g., web- or smartphone-based applications, video-based clinical visits, text messaging, etc. [33, 34]). Chronic conditions such as $\mathrm{RD}$ require active engagement by patients outside of scheduled clinical visits to help patients understand and monitor their disease state between visits and recognize important symptoms [32]. Remote monitoring can help bridge the data gap between patient visits and provide clinicians with a wealth of patient-centric information quickly by allowing clinicians to have a responsive disease-management plan, resulting in better treatment outcomes. Access to clinicians was identified as a key QoC attribute, and remote monitoring in 
the form of telemedicine visits or text-based communication can improve such access, especially for patients with limited access to physical clinical visits (e.g., those living in rural areas). Between-visit monitoring of patients in remission can free up valuable time and resources that can be spent on patients experiencing acute symptoms or an increased burden of the disease [32]. Research has shown that patients' self-assessment of RD activity using remote monitoring methods correlates well with disease activity as assessed by clinicians [35]; such assessment and monitoring of the disease state can result in improved health outcomes, including higher remission rates in a shorter timeframe [36, 37]. Remote monitoring can increase accessibility and clinician engagement with patients, which can subsequently promote better overall health outcomes, including reduced hospitalization, improved treatment adherence, and reduced financial burdens on both individuals and the healthcare system as a whole $[37,38]$.

While there are benefits to remote monitoring, there are potential barriers to its use, such as patient access to, and their ability to use, such technology. Without a working knowledge of the technology involved in remote monitoring, the utility of and compliance with remote monitoring are limited. Training with patients and their clinicians may be required to maximize the effectiveness of such techniques, particularly among older patients. However, patients are increasingly keen to incorporate novel technology into their disease management regimens [39]. By contrast, stakeholders may be reluctant to implement remote monitoring technologies due to the costs associated with developing and implementing such systems. Further research into the short- and longterm benefits of this emerging practice for patients, clinicians, and the healthcare system as a whole may provide further insight into the benefits of investing in and promoting remote monitoring. Future efforts should focus on identifying solutions to existing and potential barriers to effective remote monitoring in order to maximize the benefits of such remote monitoring to QoC.
Discussion of QoC was conducted in an idealized manner without consideration of all contextual factors of real treatment decisions. The MDP did not allow for reflection on the ways in which QoC attributes may intersect, as clinicians attempt to devise the most advantageous treatment strategies for individual patients. However, this study did provide a useful basis for future research. The European focus of the panel may also have influenced the results; the relative importance of costs related to treatment and decision-making may be different in a non-European context [40]. In addition, only the perspective of clinicians was elicited and evaluated; to obtain a holistic view of QoC, research should explore the perspectives of other stakeholders such as patients with $\mathrm{RD}$, patient advocacy groups, and payers.

\section{CONCLUSIONS}

In conclusion, this study achieved clinician consensus and has developed a framework in which to understand the attributes that constitute QoC in RD. Quality of care is a complex concept; future work can build on the presented framework to improve the lives of patients, equip clinicians with tools to provide greater QoC, and improve the healthcare system overall.

\section{ACKNOWLEDGEMENTS}

Funding. The project was funded by Merck \& Co., Inc. (Kenilworth, NJ, USA), as were all article processing charges. All authors had full access to all of the data in this study and take complete responsibility for the integrity of the data and accuracy of the data analysis.

Authorship. The authors meet the criteria for authorship as recommended by the International Committee of Medical Journal Editors (ICMJE), were fully responsible for all aspects of manuscript development, and approved this manuscript for submission. 
Medical Writing and/or Editorial Assistance. This project is a collaboration that includes Merck \& Co. scientists, academic researchers/clinicians, and Adelphi Values. The authors gratefully acknowledge Joachim Sieper, Gerd Burmester, Annelies Boonen, Loreto Carmona, Nemanja Damjanov, Valeria Rios Rodriguez, Nagy Gyorgy, Patrick Durez, Dimitrios Vassilopoulos, Antoni Chan, Karel Pavelka, Estibaliz Loza, and Esperanza Naredo for their participation.

Disclosures. Sumesh Kachroo is an employee of Merck Sharp \& Dohme Corp., a subsidiary of Merck \& Co., Inc. (Kenilworth, NJ, USA), and holds stock and options. Merck participated in the interpretation of data, review, and approval of the publication. Finbar O'Shea has received grants and fees for participation in advisory boards from Mundipharma, Sanofi, Roche, UCB, Janssen, Novartis, Astra Zeneca, and Cellgene. Finbar $\mathrm{O}$ 'Shea has received grants and fees for participation in speaker events from Pfizer, AbbVie, and Merck Sharp \& Dohme Corp., and honoraria from Merck for participation in a modified Delphi panel. Finbar O'Shea is an employee of St. James's Hospital, Dublin, Ireland. Peter C Taylor has received grants and personal fees from Eli Lilly and Company, Galapagos, and UCB. He has received grants from Abide Therapeutics and Celgene, and personal fees from AbbVie, Biogen, GSK, Janssen, Sanofi, Novartis, Pfizer, and Sandoz. He has received honoraria from Merck for participation in a modified Delphi panel. Peter C Taylor is an employee of the University of Oxford. Gavin Dickie is an employee of Adelphi Values, LLC and was a paid consultant of Merck Sharp \& Dohme Corp., a subsidiary of Merck \& Co., Inc. (Kenilworth, NJ, USA). Andrew Yaworksy is an employee of Adelphi Values, LLC and was a paid consultant of Merck Sharp \& Dohme Corp., a subsidiary of Merck \& Co., Inc. (Kenilworth, NJ, USA). Benjamin Banderas is an employee of Adelphi Values, LLC and was a paid consultant of Merck Sharp \& Dohme Corp., a subsidiary of Merck \& Co., Inc. (Kenilworth, NJ, USA). Sumesh Kachroo is an employee of Merck Sharp \& Dohme Corp., a subsidiary of Merck \& Co., Inc. (Kenilworth, NJ, USA), and holds stock and options.
Compliance with Ethics Guidelines. As this study involved expert participants providing information on QoC in rheumatic disease, rather than information on themselves, independent review board approval was not deemed necessary. However, experts provided consent before participation, in line with best practice.

Data Availability. The datasets analyzed during the current study are available from the corresponding author on reasonable request.

Open Access. This article is distributed under the terms of the Creative Commons Attribution-NonCommercial 4.0 International License (http://creativecommons.org/licenses/ by-nc/4.0/), which permits any noncommercial use, distribution, and reproduction in any medium, provided you give appropriate credit to the original author(s) and the source, provide a link to the Creative Commons license, and indicate if changes were made.

\section{REFERENCES}

1. Guidelines for the management of rheumatoid arthritis. 2002 Update. Arthritis Rheum. 2002;46(2):328-46.

2. Braun J, Sieper J. Ankylosing spondylitis. Lancet. 2007;369(9570):1379-90.

3. Aletaha D, Neogi T, Silman A, Funovits J, Felson D, Bingham C, et al. 2010 Rheumatoid arthritis classification criteria: an American College of Rheumatology/European League Against Rheumatism collaborative initiative. Arthritis Rheum. 2010;62(9):2569-81.

4. Amherd-Hoekstra A, Naher H, Lorenz HM, Enk AH. Psoriatic arthritis: a review. JDDG. 2010;8(5):332-9.

5. Sevrain M, Villani AP, Rouzaud M, Barnetche T, Paul C, Richard MA, et al. Treatment (biotherapy excluded) of psoriatic arthritis: an appraisal of methodological quality of international guidelines. JEADV. 2014;28(Suppl 5):33-9.

6. Husted JA, Gladman DD, Farewell VT, Cook RJ. Health-related quality of life of patients with psoriatic arthritis: a comparison with patients with rheumatoid arthritis. Arthritis Rheum. 2001;45(2):151-8. 
7. van Genderen S, van den Borne C, Geusens P, van der Linden S, Boonen A, Plasqui G. Physical functioning in patients with ankylosing spondylitis: comparing approaches of experienced ability with self-reported and objectively measured physical activity. J Clin Rheumatol. 2014;20(3):133-7.

8. Sandıkçı SC, Özbalkan Z. Fatigue in rheumatic diseases. Eur J Rheumatol. 2015;2(3):109-13.

9. Kvien TK. Epidemiology and burden of illness of rheumatoid arthritis. Pharmacoeconomics. 2004;22(2 Suppl 1):1-12.

10. Martindale J, Shukla R, Goodacre J. The impact of ankylosing spondylitis/axial spondyloarthritis on work productivity. Best Pract Res Clin Rheumatol. 2015;29(3):512-23.

11. Haroon N, Kim TH, Inman RD. NSAIDs and radiographic progression in ankylosing spondylitis: bagging big game with small arms? Ann Rheum Dis. 2012;71(10):1593-5.

12. Lee S, Lee SW, Song Y, Sung IH, Kim TH. Intraarticular sacroiliac corticosteroid injections in ankylosing spondylitis (abstract). Arthritis Rheumatol 2015;67(Suppl 10).

13. Kumar A, Marwaha V. New therapies for rheumatoid arthritis. Med J Armed Forces India 2003;59(2):90-92.

14. Gladman DD, Antoni C, Mease P, Clegg DO, Nash P. Psoriatic arthritis: epidemiology, clinical features, course, and outcome. Ann Rheum Dis. 2005;64(2):14-7.

15. Braun J, van den Berg R, Baraliakos X, Boehm H, Burgos-Vargas R, Collantes-Estevez E, et al. 2010 update of the ASAS/EULAR recommendations for the management of ankylosing spondylitis. Ann Rheum Dis. 2011;70(6):896-904.

16. Menter A, Gottlieb A, Feldman SR, Van Voorhees $\mathrm{AS}$, Leonardi CL, Gordon $\mathrm{KB}$, et al. Guidelines of care for the management of psoriasis and psoriatic arthritis: section 1. Overview of psoriasis and guidelines of care for the treatment of psoriasis with biologics. J Am Acad Dermatol. 2008;58(5):826-50.

17. Singh JA, Saag KG, Bridges SL Jr, Akl EA, Bannuru RR, Sullivan MC, et al. 2015 American College of Rheumatology guideline for the treatment of rheumatoid arthritis. Arthritis Care Res (Hoboken). 2016;68(1):1-25.

18. World Health Organization. Quality of care: a process for making strategic choices in health systems. Geneva: WHO; 2006.
19. Fautrel B, Guillemin F. Cost of illness studies in rheumatic diseases. Curr Opin Rheumatol. 2002;14(2):121-6.

20. van den Akker-van Marle ME, Chorus AM, Vliet Vlieland TP, van den Hout WB. Cost of rheumatic disorders in the Netherlands. Best Pract Res Clin Rheumatol. 2012;26(5):721-31.

21. Office of Behavioral \& Social Sciences Research. Defining quality of care 2016. Available from: http:// www.esourceresearch.org/eSourceBook/Evaluating theQualityofHealthCare/3DefiningQualityofCare/ tabid/797/Default.aspx\#LiveContent[Sec3Ref1. Accessed 15 March 2016.

22. Aletaha D, Alasti F, Smolen JS. Chronicity of rheumatoid arthritis affects the responsiveness of physical function, but not of disease activity measures in rheumatoid arthritis clinical trials. Ann Rheum Dis. 2015;74(3):532-7.

23. Smolen JS, Breedveld FC, Burmester GR, Bykerk V, Dougados M, Emery P, et al. Treating rheumatoid arthritis to target: 2014 update of the recommendations of an international task force. Ann Rheum Dis. 2016;75(1):3-15.

24. Carr A, Hewlett S, Hughes R, Mitchell H, Ryan S, Carr $\mathrm{M}$, et al. Rheumatology outcomes: the patient's perspective. J Rheumatol. 2003;30(4):880-3.

25. MacLean $\mathrm{CH}$, Louie $\mathrm{R}$, Leake B, McCaffrey DF, Paulus HE, Brook RH, et al. Quality of care for patients with rheumatoid arthritis. JAMA. 2000;284(8):984-92.

26. Sixma HJ, Kerssens JJ, Campen CV, Peters L. Quality of care from the patients' perspective: from theoretical concept to a new measuring instrument. Health Expect. 1998;1(2):82-95.

27. Jacobi CE, Boshuizen HC, Rupp I, Dinant HJ, van den Bos GA. Quality of rheumatoid arthritis care: the patient's perspective. Int J Qual Health Care. 2004;16(1):73-81.

28. Dalkey N, Helmer O. An experimental application of the Delphi method to the use of experts. Manage Sci. 1963;9(3):458-67.

29. Kappelman MD, Palmer L, Boyle BM, Rubin DT. Quality of care in inflammatory bowel disease: a review and discussion. Inflamm Bowel Dis. 2010;16(1):125-33.

30. Brook RH, McGlynn EA, Shekelle PG. Defining and measuring quality of care: a perspective from US researchers. Int $\mathrm{J}$ Qualit Health Care. 2000;12(4):281-95. 
31. Donabedian A. The quality of care. How can it be assessed? JAMA. 1988;260(12):1743-8.

32. Celler BG, Lovell NH, Basilakis J. Using information technology to improve the management of chronic disease. Med J Aust. 2003;179(5):242-6.

33. Baumgart DC. Personal digital assistants in health care: experienced clinicians in the palm of your hand? Lancet. 2005;366(9492):1210-22.

34. Grainger R, Townsley H. Apps for people with rheumatoid arthritis to monitor their disease activity: a review of apps for best practice and quality. JMIR Mhealth Uhealth. 2017;5(2):e7.

35. van Riel P, Alten R, Combe B, Abdulganieva D, Bousquet $\mathrm{P}$, Courtenay $\mathrm{M}$, et al. Improving inflammatory arthritis management through tighter monitoring of patients and the use of innovative electronic tools. RMD Open. 2016;2(2):e000302.

36. Salaffi F, Carotti M, Ciapetti A, Di Carlo M, Gasparini S, Farah S, et al. Effectiveness of a telemonitoring intensive strategy in early rheumatoid arthritis: comparison with the conventional management approach. BMC Musculoskelet Disord. 2016;17(1):146.

37. El Miedany Y, El Gaafary M, Youssef S, Bahlas S, Almedany S, Ahmed I, et al. Toward electronic health recording: evaluation of electronic patientreported outcome measures system for remote monitoring of early rheumatoid arthritis. J Rheumatol. 2016;43(12):2106-12.

38. Jayasena R, Cellar B, Sparks R, Varnfield M, Li J, Nepal S. Monitoring of chronic disease in the community: Australian telehealth study on organisational challenges and economic impact. Int $\mathrm{J}$ Integr Care. 2016;16(6):A106.

39. Omer T. Empowered citizen 'health hackers' who are not waiting. BMC Med. 2016;14(1):118.

40. Ridic G, Gleason S, Ridic O. Comparisons of health care systems in the United States, Germany and Canada. Mater Sociomed. 2012;24(2):112-20. 